\title{
XXV. Über die Pyroëlektricität anscheinend centrisch-symmetrischer Krystalle und über die Anomalien der Alaune und des Topases.
}

\author{
Von \\ J. Beckenkamp in Würzburg.
}

(Mit 6 Teztfiguren.)

I.

Z̈ber centrisch-symmetrische Pyroölektricitït.

W. Voigt bemerkt ${ }^{1}$ ) in bezug auf meine Untersuchungen von centrischsymmetrischen Krystallen:

"Beobachtungen von Beckenkamp an Aragonit und Baryt nach der Kundtschen Methode stimmen in ihren Resultalen im allgemeinen mit den von II ankel erhaltenen überein." "Wiederholt hat sich Beckenkamp mit der Erklärung der Pyroëlektricität überhaupt und derjenigen der centrischen Wirkungen insbesondere beschäftigt «, wesentlich ist allein, daß er alle Pyroëlektricität im Grunde als polar betrachtet und überall, wo er dergleichen an scheinbar holoëdrischen Krystallen antrifft, $Z$ willingsbildung voraussetzt. So ist ihm z. B. jeder Aragonit- und Barytkrystall ein Complex von acht hemimorphen Individuen. « In einer Fußnote fügt W. Voigt hinzu: > freilich scheinen manche Betrachtungen hiermit nicht vereinbar; insbesondere operiert die Theorie in Wiedern. Ann. 1897, 61, 600 mit centrisch geladenen Molekïlen «.

Vergleichen wir damit den Wortlaut ${ }^{2}$ ) der von mir gegebenen Deutung der Beobachtungen am Baryt und Aragonit.

- Den Beobachtungen entsprechend haben wir für einen sehr kleinen Würfel, dessen Flächen den drei Pinakoiden (100), (010) und (001)

1) Verhandl. d. k. Ges. d. Wiss. zu Güttingen 1905 , S. 399 u. 400.

z) Diese Zeitschr. 1897, 28 , 41. 
parallel gehen, die allgemeinste Annahme gemacht, daß zu jeder Fläche eine normale und zwei tangentiale elektrische Krafllinien gehören. Die auf der (senkrecht zur $a$-Axe gelegenen) $+x$-Fläche zum Ausdruck kommenden elektrischen Kräfte seien $x_{x}, y_{x}, z_{x}$; auf der $+y$-Fläche seien dieselben $y_{y},{ }^{\approx} y, x_{y}$; auf der $+z$-Fläche entsprechend $x_{z}, x_{z}, y_{z}$ *

$\gg$ Nach den bisherigen Beobachtungen scheint es aber nicht ausgeschlossen, daß für die centrisch-symmetrischen Krystalle die drei Kräfle $x_{x}, y_{y}, z_{z}$ gleich Null zu setzen sind. Geschieht dies, dann bleibt der einfache Barytkrystall auch in elektrischer Hinsicht centrischsymmetrisch; d. h. jede durch das Symmetriecentrum gelegte Gerade trifft $z u$ beiden Seiten in gleichem Abstande vom Centrum gleichwertige Punkte des Krystalles. \&

Bei solchen Körpern, welche schon äußerlich hemimorph erscheinen, muß eine die polare Pyroëlektricität im gewöhnlichen Sinne bedingende normale Kraft $x_{x}$, oder $y_{y}$ oder $z_{z}$ in den Vordergrund treten.

Dieselbe Annahme, daß beide Arten, polare und centrisch-symmetrische Erregung, an einem und demselben Minerale neben einander bestehen können, macht auch Voigt. Er schreibt 1): „Die wirklich centrische Pyroëlektricität ist also - ebenso und aus ähnlichen Gründen wie die Doppelbrechung des Lichtes - eine speciell krystallographische Erscheinung, wobei obendrein das reguläre System ausfält. Sie ist natürlich keineswegs an die centrische Symmetrie gebunden und kann bei acentrischen Krystallen neben der acentrischen auftreten.*

Vielleicht unterscheidet sich meine Auffassung allerdings insofern von der.Voigts, daß ich auf Grund meiner namentlich in der letzten Zeit gesammelten Erfahrungen der Ansicht zuneige, daB allen krystallisierten Körpern nicht nur centrische, sondern auch acentrische elektrische Kräfte zukommen; daß bei den gewöhnlich centrisch-symmetrisch ausgebildeten Krystallen die ersteren, bei den gewöhnlich hemimorph ausgebildeten die letzteren graduell überwiegen. Dieser Auffassung könnte freilich entgegengehalten werden, daß die pyroëlektrische Erregbarkeit der anscheinend centrisch-symmetrischen Krystalle nicht nur von einer Mineralart zur anderen, sondern auch bei derselben Mineralart von einem Vorkommen zum anderen bedeutende Schwankungen erkennen läßt, und daß deshalb die centrisch-pyrö̈lektrische Erregbarkeit eines bestimmten Vorkommens vielleicht nur eine $\gg$ Ausnahmeerscheinung " darstelle.

Diesem Bedenken gegenüber machte ich jedoch geltend 2): ,Graduelle Unterschiede der pyroëlektrischen Erregbarkeit bestehen auch bei solchen

1) $S .417$.

2) Diese Zeitschr. 3i, 478. 
Krystallen, deren geometrische Hemimorphie außer Frage steht. Auffallend groß sind dieselben z. B. beim Quarz. "

Auch Voigt betrachtet die centrische Erregbarkeit nicht als eine Ausnahmeerscheinung. Er schreibt 1): "Angesichts der bedeutenden quantitativen Unterschiede, welche die polare elektrische Erregbarkeit des Turmalins je nach dem Vorkommen zeigt, könnten starke quantitative Verschiedenheiten der Erregbarkeit centrischer Krystalle nicht allzusehr überraschen. ^Die großen qualitativen Unterschiede bieten aber natürlich für die Deutung eine gewisse Schwierigkeit.

Diese letztere Schwierigkeit ist nach meiner Auffassung nur ein Beweis dafür, daß die betreffenden Körper zwillingsartige Aggregate darstellen, und nicht immer die gleichen Seiten nach außen kehren. Dafür spricht auch das Verhalten dünner Spaltungslamellen, auf welches ich früher hingewiesen habe ${ }^{2}$ ): „Das Vorherrschen der positiven Elektricität auf der einen Basisfläche, der negativen auf der anderen bei dünnen Spaltungslamellen folgt dann daraus, daß auf jener durch Zwillingsbildung in der Mitte die tangentialen positiven Pole zusammenstoßen, auf dieser die negativen. \&

Bezüglich der Erklärung der von mir aus den Beobachtungen nachgewiesenen centrischen Erregung bemerkte ich $\left.{ }^{3}\right): \gg d a B$ die Theorie im vorliegenden Falle keine Pyroëlektricität kennt, ist die Folge einer für diesen Fall zu engen Grundvorstellung über die elektrische Anordnung bei dem elementaren Würfel, von welcher die Fundamentalgleichungen abhängen a.

Gerade die von mir behauptete, paradox erscheinende centrische Polarität war wohl die Ursache, daB manche Fachgenossen in meinen Beobachtungen nur zufällige Anomalien sehen wollten, während andere dieselben durch die jetzt auch von Voigt zur Erklärung der pyroëlektrischen Erregungen angenommenen chemischen Beimengungen zu erklären suchten ${ }^{4}$ ).

II.

\section{Über die centrisch-symmetrische Baryt- und Aragonitmasse.}

Bezüglich meiner Behauptung, die allseitig ausgebildeten Aragonit- und Barylkrystalle seien Zwillingsaggregate, bemerkt Voigt: „Eine Stütze für diese Anschauung findet er in kleinen Dissymmetrien an Ätzfiguren bei sehr kräftigem Ätzen, - Wahrnehmungen, die von anderer Seite bestritten werden. - Hierzu muß ich bemerken: daß ein allseitig ausgebildeter Aragonit- oder Barytkrystall einen Complex aus acht Individuen darstellt,

\footnotetext{
1) S. 397.

2) Diese Zeilschr. 28, 92.

3) Ebenda 28, 90.

4) Ebenda 84, 477 .
} 
ist nicht Hypothese, sondern unmittelbare Beobachtung. Ebensowenig beruht die von mir behauptete übereinstimmende centrische, aber nicht rhombisch-holoëdrische Symmetrie der Pyroëlektricität und der Ätzfiguren der homogenen Partien von Aragonit und Baryt auf einer Hypothese, sondern auf unmiltelbarer Beobachtung. Eine nicht zu rechtfertigende Hypothese abcr ist es, für dichomogene Masse der einfachen Individuen die Symmetrie der Oberfläche des ganzen Complexes zu postulieren. Dieser zerfällt sowohl infolge der Anwachspyramiden als auch infolge der Ätzfiguren in verschiedene Teile.

Ich selbst habe die Ätzfiguren an mehreren hundert Präparaten, und zwar immer in der gleichen GesetzmäBigkeit, beobachtet, und dabei unterschieden zwischen normalen Ätzfiguren, welche der Symmetrie der rhombischen Holoëdrie entsprechen, und anomalen 1), welche mit dieser nicht übereinstimmen. Die ersteren habe ich auf die Anordnung der Moleküle, die letzteren auf die elektrischen Ladungen der Moleküle zurückgeführt ${ }^{2}$. Genau in der gleichen Weise wurden die anomalen Ätzfiguren am Aragonit später beschrieben von F. Westhoff ${ }^{3}$ ) und Viola ${ }^{4}$ ). Am Baryt wurden unmittelbar nach meiner ersten Mitteilung darüber von J. Valentin ${ }^{5}$ ) natürliche, also wohl sicher nicht durch »sehr kräftiges Ätzen « hervorgebrachte, anomale Ätzfiguren beobachtet, desgleichen etwas später von E. Sommerfeld $\left.t^{6}\right)$. Alle diese Beobachtungen lassen dieselbe Felderteilung erkennen.

$\mathrm{Da} ß$ sowohl bei den künstlichen als bei den natürlichen Ätzfiguren von Baryt und namentlich von Aragonit die normalen Figuren bedeutend überwiegen, und letztere oft nur sehr schwierig oder gar nicht zu erkennen sind, hängt damit zusammen, daß bei beiden Mineralien mehr die Verteilung der Massenpunkte im Raume zur Geltung kommt, während z. B. beim Quarz die elektrische Ladung der Moleküle bedeutend größeren Einfluß auf die Ätzfiguren hat.

Daß tatsächlich die Aragonitgruppe nicht rhombisch-holoëdrische Symmetrie besitzt, bestätigt ferner das von mir beschriebene Vorkommen von Strontianit am Kaiserstuhl 7). Ein Teil der Krystalle ist spießig ausgebildet und entspricht so vollkommen dem Aragonit, daß er bis dahin für Aragonit gehalten wurde; ein anderer, ebenso häufiger Teil dagegen ist hemimorph ausgebildet. Diese Beobachtung kann nicht anders erklärt werden, als

1) Die Bezeichnung sanomal halte ich zwar nicht für entsprechend; sie mag aber der Kürze halber beibehalten $\pi \cdot e r d e n$.

2) Diese Zeitschr. 28, 94 f.

3) Vergl. diese Zeitschr. 35, 188.

4) Ebenda 30, 63.

5) Ebenda 15, 576.

6) Ebenda 34, 477.

7) Ebenda 1888, 14, 67. 
durch die Annahme, daß die ersteren Krystalle ein Zwillingsaggregat darstellen aus Individuen der letzteren Art.

Die Aggregatstructur der rings ausgebildeten Aragonit- und Barytkrystalle geht endlich ganz unzweifelhaft aus den pyroëlektrischen Beobachtungen Hankels hervor $\left.{ }^{1}\right)$. Die beiden Axenenden eines rings ausgebildeten Krystalles zeigen stets gleichnamige Elektricität. Wird aber ein prismalischer Krystall, der etwa an seinen natürlichen Enden positiv ist, durchbrochen, so erscheint am Bruchende je nach seinem $\Lambda$ bstande von den beiden Enden negative, oder schwach positive Elektricität. So kann sich ein homogener Krystall auch unter der Annahme centrisch-symmetrischer Erregung niemals verhalten. Diese Beobachtungen beweisen ohne jeden Zweifel, daß der Krystall eine Homogenitätsgrenze besitzt, also einen Zwilling darstellt. Erfolgt der Bruch in unmittelbarer Nähe der Zwillingsgrenze, dann verhalten sich die beiden natürlichen Enden entgegengesetzt wie die beiden Bruchstellen. Liegt der Bruch dem einen Ende näher als die $Z$ willingsgrenze, so hat nur dieses Ende die entgegengesetzte Elektricität von derjenigen der zugehörigen Bruchfläche.

Da $B$ ein rings ausgebildeter Aragonit-oder Barytkrystall einen zwillingsartigen Complex aus mehreren l'eilen darstellt, ist somit durch alle nur irgend möglichen Beobachtungsmethoden unzweifelhaft festgelegt.

Meine Untersuchungen ${ }^{2}$ ) über das pyroëlektrische Verhalten von Baryt (und Aragonit) führten zu folgendem Resultate: Bezeichnen wir durch Pfeilrichtungen die positiven elektrischen Kraftlinien, welche gleichzeitig auch die Richtungen angeben, nach welchen die anomalen durch $\mathrm{K}_{2} \mathrm{CO}_{3}$ erhaltenen Ätzfiguren von den normalen Figuren aus vorrücken, dann gibt das in Fig. 1 angedeutete Elementarparallelepipedon die Orientierung der tangentialen Kraftrichtungen; wenn normale Kraftrichtungen bei beiden Mineralien nicht berücksichtigt werden, dann ist das System centrisch - symmetrisch. Obwohl aber die drei Ebenen der Kraftrichtungen auf einFig. 1.

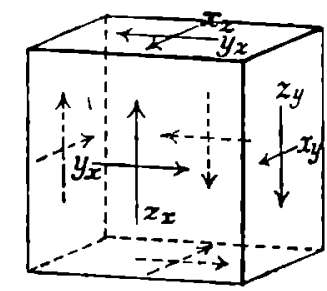
ander senkrecht stehen ${ }^{3}$, so kann das System nach der üblichen Nomenclatur nicht als rhombisch-holoëdrisch, sondern nur als striklin * bezeichnet werden, weil es weder eine Ebene, noch eine Axe der Symmetrie

1) Vergl. diese Zeitschr. 1894, 19, 252 u. 259 und 1897, 28, 78.

2) Dlese Zeitschr. 28, 78 ff.

3) Von dem in dieser Zeitschr. 36, 468 mitgeteilten Resultate, daß das Elemenlargitter des Baryts nur annähernd rhombisch ist, ist im nachfolgendon abgesehen, da diese Tatsachen hier nicht in Betracht kommen. 
besitzt. Die Anordnung dieser Parallelepipeda im Raume erfolgt jedoch nach einem der rhombischen Raumgitter. Fig. 2 zeigt die der Ebene (001) angehörenden Kräfte $x_{z}$ und $y_{z}$ und zwar sind sechs homogene Partien dargestellt, welche mit einander nach (010) und (100) verzwillingt sind.

Fig. 2.

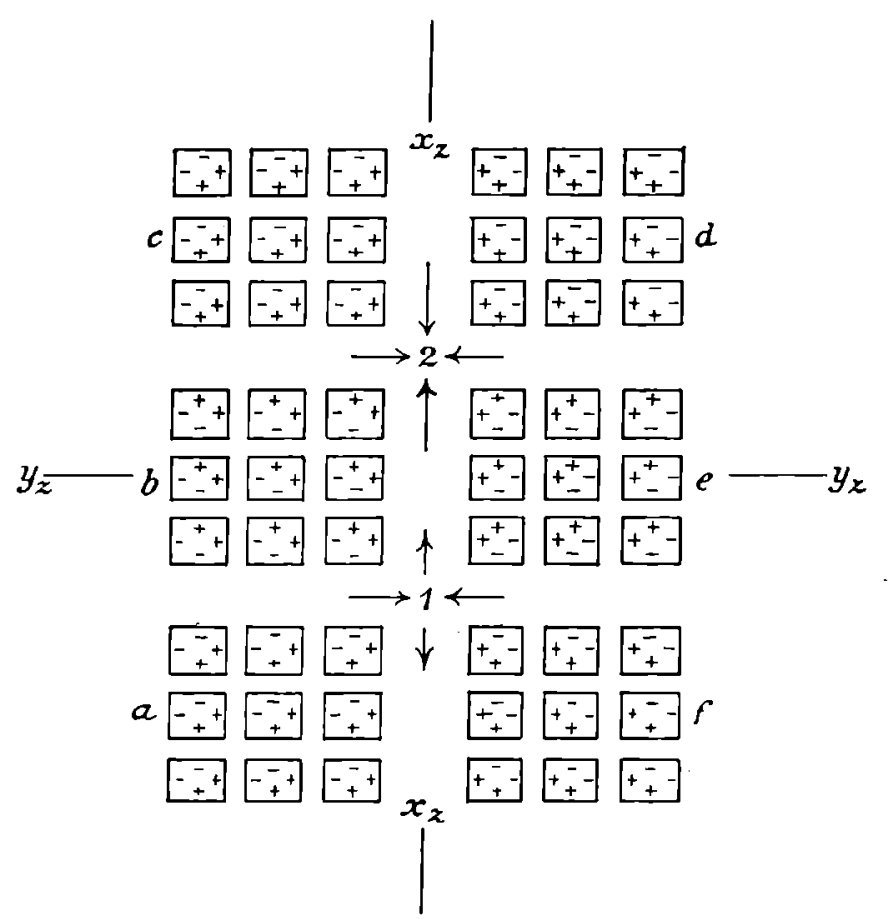

Infolge der doppelten Zwillingsbildung nach (100) ist die homogene Masse $c$ wieder in paralleler Stellung zu $a, d$ in paralleler Stellung zu $f$.

Hankel unterscheidet zwei Abteilungen von Barytkrystallen, von welchen jede nach meinen Beobachtungen aus acht Individuen besteht. Bei der ersten Abteilung legen sich vier Individuen auf (001) so an einander, wie die Massen $a, b, e, f$ um den Punkt 1; bei der zweiten Abteilung so wie die Massen $b, c, d, e$ um den Punkt 2.

Bei der ersten Abteilung tritt die elektrische Polarităt $x_{z}$ bedeutend zurück, vermutlich infolge von mehrfach wiederholter $Z$ willingsbildung nach (100) oder durch entsprechende Gruppenbildung. Infolge dieses Umstandes sind die Krystalle in der Richtung der $b$-Axe verlängert, und die anomalen Ätzfiguren folgen fast ausschließlich $y_{z}$ und $z_{y}$ (vgl. Fig. 3).

Bei der zweiten Abteilung tritt in ganz gleicher Weise infolge von mehrfach wiederholter $Z$ willingsbildung nach $(010)$ oder durch Gruppenbil- 
Uber die Pyroèlektricitat anscheinend centrisch-symmetrischer Krystalle usw. 361

dung die Polarität $y_{z}$ zurũck; diese Krystalle sind deshalb gestreckt nach der $a$-Axe, die anomalen Ätzfiguren folgen fast ausschließlich den Kraftrichtungen $x_{z}$ und $z_{x}$ (Fig. 4.).

Fig. 3.

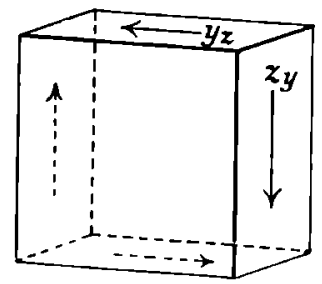

Fig. 4 .

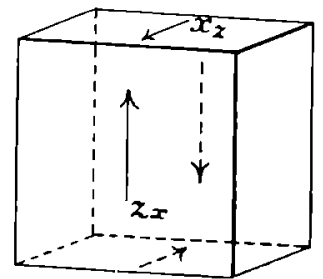

Beim Aragonit habe ich zuerst ganz analog nur anomale Ätzfiguren nach $x_{z}$ und $z_{x}$ beobachtet; die von mir untersuchten Krystalle entsprachen also der zweiten Abteilung des Baryts. Nach den Angaben 1) von v. Ebner über seine nur an ganzen Aragonitkrystallen beobachteten Ätzfiguren muß dessen Material dagegen Ätzfiguren nach $y_{z}$ und $z_{y}$ geliefert haben, entsprechend der ersten Abteilung des Baryts.

Eine vollständige Trennung der beiden Abteilungen besteht zwar nicht, jedoch beobachtete ich an einem Krystalle der einen Art immer nur ganz wenige und kleine Partien der anderen Art.

Auf Grund der Ätzfiguren sollte man hiernach den Baryt und den Aragonit für monoklin halten; und tatsächlich hat Viola ${ }^{2}$ ) am Aragonit schöne natürliche Ätzfiguren beobachtet, infolge deren er den Aragonit in das monokline System einzuordnen veranlaßt wurde, und zwar war bei diesem Vorkommen (010) Spiegelebene; die Krystalle entsprechen somit der zweiten Abteilung des Baryts.

Die Barytkrystalle sind in der Regel an einem Ende derjenigen Axe,

Fig. 5.

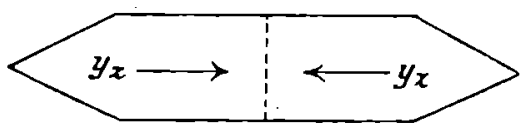

Fig. 6.

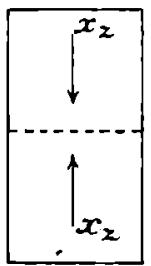

nach welcher sie verlängert sind, aufgewachsen. Sind sie in anderer Weise aufgewachsen, dann haben sie in der Regel eine Homogenitälsgrenze und

1) Vgl. diese Zeilschr. 18, 252.

2 ' Diese Zeitschr. 28, 320 und 30, 63. 
zwar in der ersten Art nach (010) (Fig. 5), die der zweiten Art nach (100) (Fig. 6). Selten fehlen diese beiden Grenzen. Fehlen sie, dann sind die Krystalle der ersten Art an beiden Enden der $b$-Axe von verschiedenen Flächen umgrenzt. Infolge der stets vorhandenen Zwillingsbildung nach (001) zeigen die anomalen Ätzfiguren auf (001) und auf (00才) nach demselben Ende der $b$-Axe. Wenn nun die Zwillingsbildung nach (100) sich so häufig wiederholt, daß der Krystall, von der Zwillingsgrenze nach (001) abgesehen, als quasi homogener Körper betrachtet werden kann, oder wenn eine entsprechende Gruppenbildung vorliegt, dann verhält sich ein derartiger Krystall bezüglich seiner Oberfläche genau wie ein rhombischer Krystall, welcher nach der $b$-Axe hemimorph ist. Dasselbe gilt aber auch für die zweite Abteilung der Barytkrystalle in dem entsprechenden Falle bezüglich der $a$-Axe. Beide Arten von Krystallen sind verschiedentlich beobachtet worden, aber gerade der Umstand, daß man hemimorphe Krystalle nach der $b$-Axe und solche nach der $a$-Axe fand, war die Ursache, daß man dieselben als vVerzerrungen * bezeichnet hat, "welche zufälligen stürenden Einflüssen ihre Entstehung verdanken $\ll 1)$. Wollte man bei diesen Krystallen von Stürungen sprechen, so müßte man auch die gewöhnliche Ausbildungsweise, bei welcher die Krystalle an einem Ende aufgewachsen waren, als gestört bezeichnen, weil bei diesen und zwar in beiden Abteilungen, der antiloge Pol durch das Anwachsen an der Ausbildung verhindert wurde, und nur der frei herausragende analoge Pol sich entfalten konnte.

Das S. 359 erwähnte pyroëlektrische Verhalten von Bruchstücken bedarf hiernach keiner weiteren Erklärung.

\section{III.}

\section{Kinetische Erklärung der Polarität des Elementar- parallelepipedons.}

Zur Erklärung der aus der Beobachtung gewonnenen Eigenschaften des Elementarparallelepipedons ging ich von folgender Überlegung aus.

$\mathrm{DaB}$ die kinetische Theorie der festen Körper, speciell der Krystalle, als eine notwendige Ergänzung der kinetischen Theorie der Gase postuliert werden muß, dürfte wohl nicht beanstandet werden; ebensowenig, daß die kleinsten materiellen Teile der festen Körper infolge ihres Wärmeinhaltes Vibrationen, und zwar im Gegensatze zu den Gasmolekülen, in geschlossenen Bahnen ausführen müssen. Auf diesen Postulaten fußend setze ich voraus, daß auch der die kleinsten Massenteile umgebende Äther infolge der Wärmebewegung der Massenteile in geschlossenen Bahnen verlaufende Vibrationen

1) Diøse Zeitschr. 17, 329 und 27, 683. 
ausführe. Nehmen wir für diese Schwingungen im Äther in erster Annäherung elliptische Bahnen an, so verhält sich die Bahn an verschiedenen Stellen in bezug auf diese Schwingungen verschieden. In bezug auf eine einzelne Schwingung ist dic Bahn centrisch-symmetrisch, erfolgen aber mehrere elliptische Schwingungen um einen gemeinsamen Brennpunkt, so addiert sich zu der centrisch-symmetrischen Eigenschaft noch eine polare in der Richtung der größeren Axen der Ellipsen. Ich setze nun ferner voraus, daß diese kinetischen Verschiedenheiten der einzelnen Stellen der Bahn, sei es mit, sei es ohne Vermittelung von Elektronen, auch eine elektrostatische Verschiedenheit bedingen.

IV.

\section{Die Anomalien von Alaun und Topas.}

Beim Topas zeigten Friedel und Curie, daß ein Zusammenhang bestehe zwischen der optischen Felderteilung und dem pyroëlektrischen Verhalten. Die den Feldern entsprechenden Teile einer basischen Topasplatte werden acentrisch-pyroëlektrisch erregt und nur durch die centrische Gruppierung der acentrischen Teile entsteht der Schein einer centrischen Erregung. Diese Beobachtung wurde von K. Mack ${ }^{1}$ ) bestätigt, indem er fand, da $B$ jedes optisch homogene Stück elektrisch polar erregt wird, analog wie ein acentrischer Krystall. In bezug auf die M acksche Erklärung dieser Beobachtung bemerkte ich ${ }^{2}$ ): Mack läßt es allerdings unentschieden, ob dieses Verhalten durch wirkliche Zwillingsbildung oder durch Spannungen veranlaßt werde.\& Aber »dieses Verhalten läßt sich offenbar nur dadurch érklären, daß die erwähnten Krystalle wenigstens in elektrischer Hinsich: Zwillinge sind, welche ihre beim Abkühlen positiven Enden nach außen, ihre negativen Enden nach innen kehren ".

R. Brauns dagegen vertritt ${ }^{3}$ ) die Ansicht, daß beim Topas die Hauptursache der Anomalien die isomorphe Beimischung sei, welche die Schwankungen im Winkel der optischen Axen und die optischen Anomalien des Topases hervorrufta. „Daß in den durch isomorphe Beimischungen anomalen Krystallen durch die Beimischung gewisse Druck- oder Zugkräfte auftreten, welche in den zu vorhandenen Krystallfächen gehörenden Anwachskegeln wirksam sind und nach der Symmetrie dieser Flächen das optische Verhalten in dem Bezirk der zugehürigen Anwachskegel ändern. \&

W. Voigt bemerkt in bezug auf diese Frage: »In der Mackschen Arbeit wird die ganz verschiedene Symmetrie einer centrisch-optischen Anisotropie und einer polaren elektrischen Erregung nicht auseinander-

1) Diese Zeitschr. 18, 579.

9) Ebenda 1890, 17, 323 u. 324.

3) Die optischen Anomalien der Krystalle 4891, 911 und 313. 
gehalten, und derselbe Fehler findet sich auch in der Preisschrift von Brauns über optische Anomalien.* * Mack vertritt die Anschauung, daß die Flächen, in denen scheinbare Ladungen auftreten, die geometrischen Orte ausnahmsweise starker Spannungen darstellen; indessen der Deformations- oder Spannungszustand eines Volumelementes ist nach der Lehre der Elasticitätstheorie centrisch-symmetrisch, er kann also nicht in einem centrisch-symmetrischen Krystalle einen polaren Zustand hervorrufen. In der Ansicht, daß die pyroëlektrische Erregung des Topases nicht durch Spannungen erklärt werden kann, stimmt also Voigt mit der von mir ausgesprochenen Ansicht überein. Bezüglich meiner weiteren Folgerung jedoch, daß die Erscheinungen am T'opas als $Z$ willingsbildung zu deuten seien, macht Voigt geltend, $\mathrm{da}^{\mathrm{B}}$ die heterogenen Teile des Topases in ihrer gegenseitigen Orientierung nur um wenige Grade von einander abweichen, und direct neben solchen, deren optische Symmetrie der Krystallform widerspricht, solche liegen, für die beide übereinstimmen a.

Nach Voigt müßte man somit an secundär erhaltene Polarität denken, und da physikalische Ursachen nach der oigtschen Ansicht (S. 409) kaum in Betracht kommen, so blieben für ihn nur chemische übrig. Bezüglich der Einwirkung fremder chemischer Beimengungen unterscheidet Voigt "zwei fundamental verschiedene Möglichkeiten a, entweder könnte die Beimengung das Krystallmolekül selbst verändern, oder sie könnte als feste Lösung außerhalb des Moleküls bleibend dessen Polarität bedingen. Derartige Ansichten wurden bezüglich der Erklärung der anomalen Ätzfiguren zuerst von Westh off und später von E. Sommerfeldt vertreten. Ich habe dagegen eingewendet 1 , $>$ daß keine Beobachtung eine derartige Hypothese rechtfertigt, daß vielmehr die bekannten Beobachtungen derselben direct widersprechen, indem die aufgenommenen Farbstoffe sich stets der Symmetrie des Wirtes anschließen $\propto$.

Dieser Tatsache scheint zu widersprechen, daß schon Biot (1841) angibt, die Krystalle des reinen Kalithonerde-Alauns stets isotrop gefunden zu haben. F. Klocke ${ }^{2}$ ) fand diese Angabe bestätigt und beobachtete außerdem, daß „Krystalle, welche aus isomorphen Mischungen verschiedener Alaunarten bestanden, stets stark doppeltbrechend " waren. So z. B. fand er, $\gg d a ß$ verschiedene Mischungen aus Kali- und Chromalaun alle Doppelbrechung zeigen, während die Componenten beider isotrop sind *.

Die Erklärung ergibt sich nach meiner Auffassung aus folgender Betrachtung:

Nehmen wir an, die reguläre Gruppe der Alaunmoleküle bestehe in ähnlicher Weise, wie ich für den Christobalit mit großer Wahrscheinlichkeit

1) Diese 7eitschr. 32, 30 und 36, 477.

2) N. Jahrb. f. Min. usw. $1880,1,76$. 
nachgewiesen, aus 24 chemischen Molekülen, von welchen je drei über einer Oktaëderfläche liegen. Gäbe es zwei Alaunarten, deren Molekularvolumina so annähernd congruent wären, daß ein Molekül der einen Art irgend eines der anderen Art in der oktaëdrischen Gruppe zu verlreten imstande wäre, dann müßte auch die isomorphe Mischung optisch-isotrop sein. Keine der 24 Stellungen innerhalb der Oktaëdergruppe hätte dann vor der anderen einen Vorzug, und selbst wenn durch die Anpassung des nicht genau congruenten Molekularvolumens kleine Störungen der regulären Anordnung einträten, dann müßte sich die Gesamtheit der Störungen ausgleichen.

Es können indessen die Molekularvolumina zweier Alaune einander ähnlich sein, ohne daß die Congruenz so groß ist, daß das Molekül der einen $\Lambda r t$ das der anderen zu vertreten imstande ist. Beide Arten können für sich oktaëdrische Gruppen und völlig isotrope Massen liefern. Der in größerer Menge vorhandene Alaun krystallisiert bei gleicher Iüslichkeit der beiden Arten zuerst und schiebt beim Wachsen alle fremden Massen aus dem von ihm beanspruchten Raume nach außen vor sich fort 1). Diese sammeln sich vor dem wachsenden Krystalle mehr und mehr an, bis sie selbst überwiegen und auskrystallisieren. So bilden sich abwechselnd Lamellen der beiden Alaunarten parallel zu den Umgrenzungsflächen.

Sowohl die Bildung der dreigliederigen Gruppe mit genau trigonaler Axe als auch die genau reguläre Ausgleichung der Abweichung der Dimensionen der dreigliederigen Gruppe von genau regulären Verhältnissen setzt voraus, daß keines der 3 bezüglich 24 Moleküle gegenüber irgend einem der anderen eine Sonderstellung einnehme. Dies gilt aber bei den Lamellen für alle diejenigen Gruppen nicht, deren Abstand von den Lamellengrenzen nicht genügend groß im Verhältnisse zur molekularen Wirkungssphäre ist. Für solche Gruppen kann auch bei annähernd oktaëdrischer Gruppierung doch nur diejenige Symmetrie des regulären Systems bestehen, welche der Flächensymmetrie derjenigen regulären Form zukommt, deren Flächen die Lamellen parallel sind.

Die Lage und Orientierung eines Moleküls wird aber außerdem nicht nur durch die zur gleichen Gruppe gehörigen Moleküle bedingt, sondern auch durch die Netzebenen, welchen dasselbe angehört. Ist nun eine Lamelle sehr dünn, so werden diejenigen Netzlinien bei der Orientierung eines Moleküls bevorzugt, welche der Lamelle parallel gehen. Die Gruppen haben dann natürlich nicht mehr die volle regulïre Symmetrie, sondern nur noch die einer der Lamelle parallelen Fläche einer regulären Form.

Die Annahme eines mechanischen Druckes oder Zuges im

1) Vgl. J. Beckenkamp, Die Bildung der Zellenkalke. Sitzungsber. d. phys.med. Ges. zu Würzburg 4907 , Sep.-Abz. S. 27. 
Sinne von E. Reusch zur Erklärung der Doppelbrechung läßt sich weder durch den Nachweis des Bedürfnisses nach einer -Spannung \& zum Zwecke der Erklärung der Doppelbrechung noch durch den Nachweis einer eine "Spannung * nach sich ziehenden Ursache rechtfertigen.

Im Falle der optischen Anomalie durch isomorphe Beimengungen wird also die anomale Doppelbrechung nicht durch *feste Lüsung ", sondern durch lamellare Verwachsung verschiedener Krystallmassen hervorgerufen.

Der von Brauns ${ }^{1}$ beschriebene Topaskrystall besteht aus drei sich optisch verschieden verhaltenden Teilen. Der innerste Teil einer basischen Platte ist homogen und zwar rhombisch; ebenso eine kleine Parlie zu beiden Seiten der $b$-Axe, an der Grenze des zweiten gegen den dritten Teil.

An den centralen Teil schließt sich nach außen ein zweiter optisch homogener, anscheinend trikliner Teil mit einer Auslöschungsschiefe von etwa $4^{\circ}$ gegen die Diagonale an.

Der dritte T'eil, der äußerste Rand, ist oplisch nicht homogen; die Auslöschungsschiefe wächst hier von ca. $16^{\circ}-20^{\circ}$.

Bei Platten der Randteile parallel zur verticalen Axe fand bereits Brewster eine Auslöschungsschiefe von etwa $2^{0}-3^{0}$ gegen die Verlicale. Die dem Bereiche der Prismenflächen angehörenden Partien besitzen also, wie übrigens auch Brauns hervorhebt, nicht die den Prismenflächen zukommende Symmetrie, und deshalb muß man wohl annehmen, daß der Doppelbrechung des Topases noch andere Ursachen zugrunde liegen als für den Alaun angegeben wurde.

Der letzte Teil der Topasmasse hat sich offenbar unter nicht mehr constanten Verhältnissen der Zusammensetzung des äußeren, das Material für den Krystall liefernden Mediums gebildet, und dadurch entstanden nach und nach Krystallmassen mit zunehmender Auslöschungsschiefe.

Der Umstand, daß die trikline Modification in ihren Dimensionen rhombischen Verhältnissen sehr nahe steht, bedingt zunächst die Zwillingsbildung mit rhombischer Mimesie, wie wir sie am besten im zweiten Teile, aber auch im dritten Teile erkennen. In zweiter Linie bedingt aber dieselbe Annäherung an rhombische Dimensionen auch, daß sich dem vorigen Zwillingsgeselze entsprechende Molekulargruppen von genau rhombischer Symmetrie bilden. Bei der ersten krystallinischen Ausscheidung werden sich infolge des Bestrebens der gleichmäßigen Abrundung gegen das äußere Medium ${ }^{2}$ ) nur genau rhombische Gruppen bilden. Mit der Zunahme der Oberflächengrüße wächst jedoch der orientierende Einfluß derjenigen Moleküle, welche in den betreffenden Oktauten auf der äußeren Seite der

1) Optische Anomalien S. 309, Tafel 5, Fig, 134.

2) Vgl. diese Zeitschr. 36, 487. 
Gruppe liegen, so daß schließlich die Gruppenbildung ihren rhombischen Charakter verliert. DaB dieser im vorliegenden Falle nicht etwa allein infolge einer mit der Zeit eintretenden Veränderung des Lüsungsmittels gestört wird, verrät der Umstand, daß er auch im Gebiete der zweiten Zone auftritt, und zwar auch hier nur da, wo während der Ablagerung dieser rhombischen Partie eine kleine Fläche (010) existierte. Durch diese ging eine Homogenitätsgrenze, und durch die gleichzeitige Einwirkung der zu beiden Seiten dieser Grenze liegenden, verschieden orientierten Moleküle wurde die Gruppenbildung ebenso begünstigt wie im Centrum, wo ebenfalls verschieden orientierte Moleküle nahe bei einander liegen.

V.

\section{Über die Mallardsche Theorie und die Theorie der Molekular- gruppen oder der physikalischen Moleküle.}

Voigt spricht sich, wie schon erwähnt, bezüglich des Topases gegen die Annahme eines zwillingsartigen Aufbaues aus Individuen von niedrigerer Symmetrie aus. Er schreibt: "So wenig also principiell die Existenz weiterer Analoga zu Boracit und Prehnit zu bestreiten ist, so kann man doch kaum einen anderen centrisch erregbaren und nicht anderweit als $Z$ willing. erwiesenen Krystall mit Sicherheit als ein solches bezeichnen. \& ${ }^{1}$ )

Jedoch muß Voigt zugestehen, daß die Marbachsche Deutung (1855) der optischen Anomalien, die später auch von Klocke und namentlich von $C$. Klein und seiner Schule adoptiert wurde, es trete pvielleicht bei der Bildung größerer Krystalle durch den fortschreitenden Act der Krystallisation eine Spannung der Teile ein, welche in bestimmten Schichten ihre größten Werte annimmt und dort eine merkliche Doppelbrechung und damit eine Wirkung auf polarisiertes Licht bedingt ${ }^{2}$ ), für den Topas versage. Zudem ist, wie aus vorstehenden Angaben hervorgeht, für den zwillingsartigen Aufbau von Baryt und Aragonit durchaus eindeutiges Beweismaterial in erdrückendem Maße von mir schon seit vielen Jahren nachgewiesen. Voigt begründet seinen Standpunkt in der alten, noch von Klocke herrührenden Weise.

Klocke bemerkte 3): „Die optischen Untersuchungen E. Mallards haben eine Incongruenz der morphologischen und der optischen Eigenschaften vieler Krystalle in einem über Erwarten großen Umfange constatiert. Die von Mallard zur Erklärung dieser Erscheinung aufgestellte Hypothese: jene Krystalle seien aus mehreren Individuen von niedrigerer

1) S. 408 .

z) Pogg. Ann. d. Phys. 185;, 94, 42:5.

3) N. Jahrb. l. Min. usw. 1880, 1, 53 . 
Symmetrie, als durch die Form des ganzen Krystalles ausgedrückt sei, zusammengesetzt, würde eine so einschneidende Umgestaltung unserer bisherigen krystallographischen Betrachtungsweise bedingen, daß sie zu einer allseitigen vorsichtigen Prüfung auffordert.

In gleichem Sinne schreibt Brauns): "Auf anderer Seite (Beckenkamp) hat man sich durch asymmetrische, am Baryt, Aragonit usw. beobachtete Ätzfiguren zu der Annahme verleiten lassen, daß die Moleküle dieser Mineralien asymmetrisch seien, ihre Anordnung aber nach der Symmetrie des rhombischen Systems erfolge. Würde man in dieser Weise die Form der Ätzfiguren unter allen Umständen als ausschlaggebend ansehen, so würde es bald dahin kommen, daß man nur noch asymmetrische Krystalle kennt. *

In gleichem Sinne ist wohl auch die Bemerkung von Voigt zu verstehen ${ }^{2}$ ): sEine Anschauung, die mit Ideen von Mallard u. a. verwandt, aber der sonst in der Krystallphysik mit größtem Erfolge benutzten diametral entgegengesetzt ist und jedenfalls in dieser schroffen Form sehr hedenklich ist.

Sehen wir demnach von anderen, hier nicht zu erörternden Motiven $a b$, so finden wir von Anfang an bis auf Voigt als innersten Grund für die Ablehnung der Mallardschen Ideen dieselbe Befürchtung: für die Krystallsysteme wurde von Weiß, für die Meroëdrien namentlich von Naumann die Existenzberechtigung nachgewiesen. Dazu kam die mathematisch unangreifbare Ableitung der 32 Symmetrieklassen mit ihrer vortrefflichen Gelegenheit zu weiteren mathematisch-physikalischen Deductionen. Und nun sollen auf einmal 31 von den 32 Klassen gar nicht vorkommen und ihr bisheriger Nachweis auf Täuschung beruhen?

Die Mallardsche Theorie war allerdings in ihrer speciellen Ausführung nicht einwandfrei. Die von mir infolgedessen aufgestellte Theorie der Molekulargruppen ist jedoch frei von diesen Einwänden und auch frei von der schroffen Form der Mallardschen Theorie. Nach der von mir aufgestellten Theorie existieren die Weißschen Systeme auch für streng bomogene Massen. Für »quasi homogene * Massen, welche für manche Untersuchungsmethoden auch als homogene gelten können, werden sich im großen und ganzen auch die wichtigsten Unterabteilungen wieder construieren lassen. Bemerkenswert aber bleibt, daß auch die quasi homogenen Massen sich in der Regel zu Zwillingen mit anscheinend noch höherer Symmetrie aggregieren. Da diese Aggregate Ergänzungszwillinge darstellen, so ist deren Heterogenität optisch nicht immer nachweisbar, aber bei anderen Methoden, namentlich bei Ätzliguren, bei pyroëlektrischen und bei magne-

1) Opt. Anom. S. 56 .

2) S. 399. 
tischen Untersuchungen künnen diese pseudosymmetrischen Gebilde nicht als homogene Körper behandelt werden.

Das für alle Arten von Zwillingsbildungen maßgebende Princip wurde in meiner Arbeit "Über die Dioxyde der vierten Gruppe " abgeleitet 1 ).

Die in Verbindung mit dieser Theorie der Gruppenbildung aufgebaute kinetische Krystalltheorie ruht auf einwandfreier Grundlage; ihre bisherigen IResultate sind nicht nur für die Mineralogie, sondern auch für die Physik und Chemie von gleicher Bedeutung. Leider haben die Vertreter der beiden letzteren Disciplinen im allgemeinen nur mehr wenig Fühlung mit der krystallographischen Lileratur, so daß es für die Mehrzahl derselben schwierig sein wird, den betreffenden Ausführungen zu folgen und vor allem die Beweiskraft derselben auf Grund eines objectiven Studiums der Originalarbeiten selbst zu beurteilen. Die Arbeiten Fresnels und Mitscherlichs haben schon vor vielen Jahrzehnten den Wert der Krystallographie für den Forlschritt der Physik und Chemie dargetan und es kann nicht fehlen, daß ein weiterer Ausbau der kinetischen Krystallographie das verloren gegangene Interesse wieder zurückrufen wird.

1) Diese Zeitschr. 42, 469. 